\title{
Induction of a therapeutic antitumor immunological response by intratumoral injection of genetically engineered Semliki Forest virus to produce interleukin-12
}

\author{
Ryuya Yamanaka, M.D, Susan A. Zullo, Ph.D., Ryuichi Tanaka, M.D., \\ Jay Ramsey, M.D., Michael Blaese, M.D., and Kleanthis G. Xanthopoulos, Ph.D.
}

Clinical Gene Therapy Branch, National Human Genome Research Institute, National Institutes of Health, Bethesda, Maryland; and Department of Neurosurgery, Brain Research Institute, Niigata University, Niigata, Japan

\begin{abstract}
Object. The authors investigated immunogene therapy for malignant glioma to determine whether its therapeutic efficacy could be improved.

Methods. Four groups of 203-glioma-bearing mice were treated with injections of phosphate-buffered saline, Semliki Forest virus (SFV)-LacZ, retrovirus vector DFG-interleukin (IL)-12, and SFV-IL12, respectively.

The results indicated that therapeutic immunization with SFV-IL12 prolonged the survival of mice with established tumors. Semliki Forest virus induces apoptotic death to glioma cells, which facilitates the uptake of apoptotic cells by dendritic cells, providing a potential mechanism for enhanced immunogenicity.

Conclusions. Immunogene therapy with IL-12 via SFV may be an excellent candidate for the development of new cancer vaccines.
\end{abstract}

\section{KeY WORDS • interleukin-12 • Semliki Forest virus • apoptosis \\ - immunogene therapy $\bullet$ malignant neoplasm $\bullet$ glioma}

The prognosis for patients with malignant glioma has not improved much over the past years. Treatments, including surgery, radiotherapy, and chemotherapy have not changed the prognosis for a patient with these malignant neoplasms. Therefore, the development of novel therapies for malignant glioma is essential. The CNS is an immunologically privileged region, considered to lack lymphoid reactivity and normal immune surveillance. Recently, several groups have reported immunogene therapy protocols in which they used intradermal vaccines of genetically modified tumor cells; the treatment was shown to be effective in rodent brain tumor models..$^{9,23}$

Interleukin-12 is a heterodimeric protein consisting of two subunits (p35 and p40) and is secreted by antigen-presenting cells such as DCs and macrophages. ${ }^{10,28}$ It enhances the proliferation and cytolytic functions of $\mathrm{T}$ cells and NK cells and promotes these effector cells to produce a number of other cytokines, including IFN- $\gamma .{ }^{4}$ Furthermore, IL-12 directs the differentiation of uncommitted T cells toward the Thelper type $1,{ }^{12}$ which is critical for cellmediated antitumor immunity. The antitumor effects of

Abbreviations used in this paper: BHK = baby hamster kidney; $\mathrm{CNS}=$ central nervous system; $\mathrm{CTL}=$ cytotoxic $\mathrm{T}$ lymphocyte; $\mathrm{DC}=$ dendritic cell; ELISA = enzyme-linked immunosorbent assay; FACS = fluorescent-activated cell sorter; FCS = fetal calf serum; IL = interleukin; IFN = interferon; NK = natural killer; PBS = phosphate-buffered saline; SFV = Semliki Forest virus.
IL-12 are mediated by activation of CTLs as well as NK cells $^{5,15,30}$ and by the induction of IFN- $\gamma$ production by T cells and NK cells. ${ }^{3,17}$ Recently, several investigators have reported potent antitumor effects of IL-12 delivery by using $I L-12$ gene-modified tumor cells or systemic administration of IL-12 protein. ${ }^{4,17,25}$ Based on these results, clinical trials of $I L-12$ gene therapy in which autologous fibroblasts were used have been completed. ${ }^{27}$ Partial response was observed in patients with several types of cancer. Delivery to the tumor site of $I L-12$ genes that are genetically modified to express gene products that enhance their ability to elicit a response may avoid the requirement for selective recruitment and local activation, which are disordered within the tumor microenvironment. We have observed that IL-12 production on DCs is suppressed in patients with malignant glioma (unpublished data); therefore, we suspect that one of the mechanisms of immunosuppressive properties in malignant glioma patients is IL-12 dysfunction, resulting in failure to promote and maintain antigen-specific $\mathrm{T}$ cells. To enhance the immune response further, we evaluated transduction of $I L-12$ genes in a murine brain tumors model. We investigated whether $I L-12$ gene transduction into tumor may serve a possible strategy to treat malignant glioma.

Viral vectors commonly used for therapeutic DNA delivery include replication-deficient forms of adenovirus and retrovirus. Although retroviruses have the advantage of mediating stable gene transfer with a low potential for 
immunogenicity, this vector delivery system has several therapy-related problems including difficulty in producing high titers of retrovirus, the fact that only actively dividing cells are capable of being infected, and the possibility of insertional mutagenesis. ${ }^{16}$ Whereas the adenovirus vector system is capable of delivering genes with high efficiency to a wide spectrum of nondividing cells in vivo, ${ }^{7}$ unfortunately, it has produced only transient expression with different gene products. Transient expression may result from a strong immune response of host cells against the adenovirus. ${ }^{8}$ The SFV, a member of the Alphavirus, has received considerable attention for use as a virusbased expression vector. The SFV expression system differs from currently available viral delivery systems in that it is an RNA virus, known to generate high levels of protein expression in vitro. The SFV is less pathogenic to humans. ${ }^{19}$ It is a self-amplifying expression vector that produces high-titered stocks of infectious particles, infects nondividing cells, and generates high-level expression of transgenes. ${ }^{24}$

We have previously reported that genetically modified DC-pulsed tumor complementary DNA via SFV markedly enhanced antitumor immune responses in murine glioma models. ${ }^{32}$ In the studies presented here we evaluated the efficacy of genetically modified SFV to produce IL12. The vaccines were studied in a model of active immunogene therapy for CNS tumors.

\section{MATERIALS AND METHODS}

\section{Tumor Cell Lines and Animal Models}

The 203-glioma cell line is derived from C57BL/6 mice. Baby hamster kidney cells were grown in minimum essential medium containing 10\% FCS. All cell lines were shown to be free from mycoplasma contamination. In all experiments we used 6 to 12-week-old female C57BL/6 mice, which were maintained in a virus-free environment and treated in accordance with the National Institutes of Health standards.

\section{Generation of SFV-IL12 Particles}

The plasmid pSFV3, pSFV3-LacZ contains an SP6 promoter, a 7-kb fragment encoding the SFV RNA replicase, and a subgenomic promoter that is bound by the RNA replicase to synthesize a large quantity of subgenomic RNA. A helper plasmid, pSFV-Helper 2, which contains the genes for the structural proteins (capsid, E3, E2, 6K, and E1)required for packaging of the viral genome, was used for construction of the recombinant envelope gene. Retrovirus vector DFG-IL12 was kindly provided by Dr. Nishioka. ${ }^{18}$ The $I L-12$ gene was amplified using DFGIL12 as a template and put into pSFV. The SFV-IL12 RNA was transcribed in vitro and capped using SP6 RNA polymerase and a capping analog. The BHK cells were transfected with SFV-IL12 RNA and helper virus RNA, respectively. For cotransfections of helper and expression RNA into BHK cells, electroporation was performed. Electroporated cells were transferred to $10 \mathrm{ml}$ of minimum essential medium containing 5\% FCS and incubated for 12 hours. Cells were then washed with PBS and incubated in $10 \mathrm{ml}$ of minimum essential medium without FCS. After 24 hours, culture supernatants were harvested, and aliquots were stored at $-80^{\circ} \mathrm{C}$. Recombinant SFV particles were produced. Retroviral particles were also generated as described elsewhere. ${ }^{18}$ Infectivity of recombinant viruses to BHK and 203 glioma was determined by transfer of the SFV and retroviral particles that can transduce the $\beta$-galactosidase gene. Viral supernatant dilutions were added to adherent cells $\left(2 \times 10^{5}\right)$ in 6-well plates. After 1 hour of incubation at $37^{\circ} \mathrm{C}$, cells were washed with PBS and incubated in growth medium at $37^{\circ} \mathrm{C}$ for 24 hours. Viral infection was evaluated by X-galactosidase staining. Briefly, cells were fixed in PBS containing $0.5 \%$ glutaraldehyde for 15 minutes followed by washing with PBS three times. Then cells were stained with PBS containing $1 \mathrm{mg} / \mathrm{ml} \mathrm{X}$-galactosidase, $5 \mathrm{mM}$ potassium ferricyanide, 5 $\mathrm{mM}$ potassium ferrocyanide, and $1 \mathrm{mM} \mathrm{MgSO}$ at $37^{\circ} \mathrm{C}$ for 2 hours. The activity of IL-12 was measured using ELISA, and biological activity to stimulate IFN- $\gamma$ production from ConA-treated splenocytes was also confirmed. The splenocytes $\left(1 \times 10^{5} / \mathrm{ml}\right)$ was stimulated by supernatant of transfectant, and the IFN- $\gamma$ production was also estimated by ELISA.

\section{In Vitro Cytotoxicity Assay}

In vitro cell-mediated cytotoxicity assays were performed using standard procedures as previously described. ${ }^{31}$ In this study, splenocytes obtained in immunized and control mice were restimulated in vitro for 5 days on monolayers of mitomycin C-treated 203-glioma cells. Target cells included 203-glioma cells.

\section{Implantation of Brain Tumors}

The glioma cells were harvested by trypsinization and washed twice in Dulbecco PBS. The cells (500 cells in a volume of $5 \mu \mathrm{L}$ ) were then implanted into the right-sided caudate nucleus of the brain of the C57BL/6 mice by stereotactic injection, as previously described. ${ }^{31}$ The mice were examined macro- and microscopically. Intratumoral cells were evaluated using anti-CD8 antibody and fluorescein isothiocyanate labeled anti-rat immunoglobulin G.

\section{Cell Death and Uptake Analysis}

The cell death and uptake analysis was performed as previously described..$^{33}$ Briefly, the self-replicating SFV was transcribed in vitro from the pSFV-Lac Z or IL-12 plasmid and then transfected into the 203-glioma cells. As a control, the plasmid pLac Z-C1 was also transfected into the 203-glioma cells. Viable cells were counted every 24 hours. In uptake experiments, 203-glioma cells were transfected with SFV-IL12 and then labeled 24 hours later with PKH26-GL. Labeled cells $\left(1 \times 10^{6}\right)$ were cocultured with $1 \times 10^{6}$ bone marrow-derived immature DCs prepared by culture of bone marrow cells for 4 days with granulocyte-macrophage colony-stimulating factor and IL-4. Cells were then stained with fluorescein isothiocyanate-labeled anti-I-A $\mathrm{A}^{\mathrm{b}}$ monoclonal antibody and analyzed by FACS.

\section{Statistical Analysis}

Survival estimates and median survivals were determined using the method of Kaplan and Meier. Survival data were compared using the Wilcoxons test. The Student t-test was used for calculating the significance of 


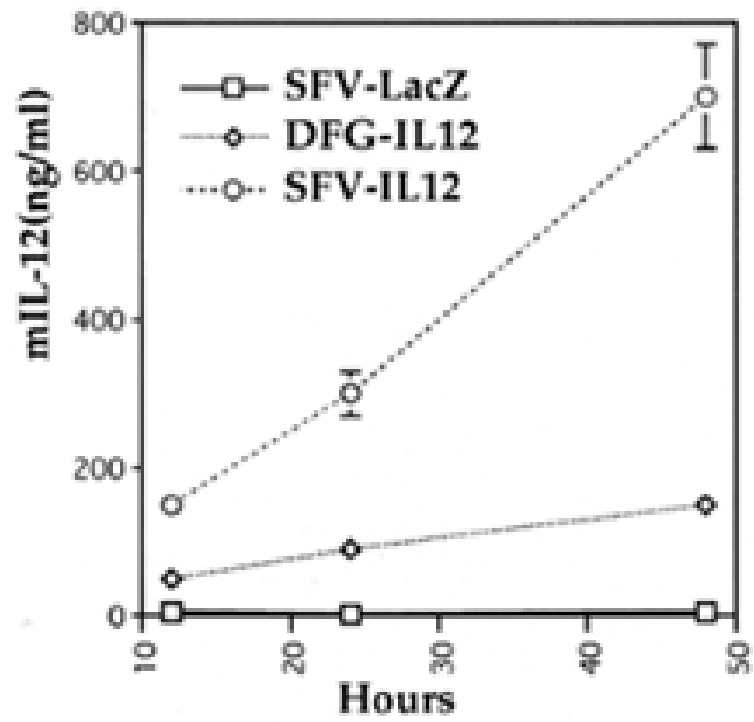

Fig. 1. Graph showing that 203-glioma cells can be efficiently transduced with an SFV-IL12 vector and produce IL-12. The production of IL-12 by $I L-12$ gene-modified glioma cell is shown. The glioma cells were transduced with DFG-IL12, SFV-LacZ, and SFV-IL12, respectively. After transduction on Day 5, supernatant in the glioma cell culture was collected and assayed in an IL-12 ELISA.

other data. Statistical significance was considered to be less than 0.05 .

\section{Sources of Supplies and Equipment}

The 203-glioma cell line was kindly provided by Riken Cell Bank (Tsukuba, Japan). The BHK cells were aquired from Invitrogen (San Diego, CA). The minimum essential medium was purchased from GIBCO-BRL, (Gaithersburg, MD). We obtained the mice from Jackson Laboratory (Bar Harbor, ME).

The plasmid pSFV3, pSFV-LacZ was aquired from Life Technologies (Gaithersburg, MD), as was the capping analog. The retrovirus vector DFG-IL12 was kindly provided by Dr. Nishioka (Department of Surgery, School of Medicine, University of Pittsburgh Cancer Institute, University of Pittsburgh) Cells were incubated in minimum essential medium obtained from GIBCO-BRL. The IL-12 activity was measured by an ELISA kit produced by R\&D systems (Tokyo, Japan). The anti-CD8 antibody and fluorescein isothiocyanate-labeled anti-rat immunoglobulin $\mathrm{G}$ were obtained from Pharingen (La Jolla, CA), as was the anti-I-A $\mathrm{A}^{\mathrm{b}}$ antibody. The XTT assay kit (Boehringer Mannheim, Tokyo, Japan) was used to count viable cells. In uptake experiments, glioma cells were transfected and later labeled with PKH26-GL obtained from Sigma Chemical Co. (St. Louis, MO).

\section{RESULTS}

\section{Dendritic Cell Generation and Infection With Recombinant Viruses}

As described previously, ${ }^{2}$ after 7 days of culture, DC aggregates were observed, and these cells were major his- tocompatibility complex class I, major histocompatibility complex class II, CD80, and CD86+, respectively. Infectivities of recombinant viruses were determined by transfer of the pSFV-lacZ, DFG-lacZ that can transduce the $\beta$-galactosidase gene. Viruses had a very high infectious titer against $\mathrm{BHK}$ cells $\left(5 \times 10^{7} \mathrm{lacZ} \mathrm{cfu} / \mathrm{ml}\right)$, and 203-glioma $\left(4 \times 10^{7} \mathrm{lacZ} \mathrm{cfu} / \mathrm{ml}\right)$. After completion of transduction procedures performed on Days 3 and 7, the concentration of IL-12 p70 heterodimer released into the culture media was measured in an ELISA. As shown in Fig. 1, accumulation of IL-12 was observed in cultures of SFV-IL12- and DFG-IL12-transduced 203-glioma cells but not in cultures of PBS- and SFV-LacZ-transduced cells. Interleukin-12 production from IL-12-transduced 203-glioma cells ranged from 150 to $180 \mathrm{ng} / 10^{6}$ cells $/ 48$ hours for DFG-IL12 and 650 to $750 \mathrm{ng} / 10^{6}$ cells/48 hours for SFV-IL12. The IL-12 protein produced by the genemodified 203-glioma cells was confirmed to be biologically active and capable of stimulating IFN- $\gamma$ production from ConA-treated splenocytes (data not shown).

\section{Prolonged Survival of Mice Bearing CNS Glioma Cells and Treated Using IL-12 via SFV}

When considering the clinical application of a tumor vaccination strategy, it is realistic to treat animals with tumor present at the time of vaccination. Thus 500 viable 203-glioma cells were implanted in the brain of naïve mice, and treatment was undertaken 7 days later. Animals were separated into groups and received three intratumoral injections spaced 1 week apart with PBS, SFVLacZ, DFG-IL12, and SFV-IL12, respectively. The titer of these virus was all $1 \times 10^{8}$ particles $/ \mathrm{ml}$. Mice in the first three groups had median survivals of 20 to 35 days. Mice treated with IL-12 via SFV had a significantly longer median survival of 90 days $(p<0.05)$; seven of 14 of these mice were alive at Day 90 when the experiment was stopped.

\section{Tumor-Specific Immune Response Identified After Treatment With SFV-IL12}

We have investigated whether intratumoral injection of IL-12 via SFV is capable of inducing tumor-specific CTLs. Standard cytotoxicity assays were performed using splenocytes harvested from treated animals 7 days after the third intratumoral injection and were restimulated for 5 days in vitro with irradiated 203-glioma cells. Cytotoxic activity was tested against glioma cells (Fig. 2). As shown in Fig. 3, SFV-IL12 immunization induced 203 glioma cell-specific CTL responses that were statistically significant compared with animals immunized using either SFV-LacZ, DFG-IL12, or PBS ( $\mathrm{p}<0.01)$.

\section{Intratumoral Infiltration of CD8 T Cells Evident in SFV- IL12-Treated Mice}

Examination of the brains of the SFV-IL12-treated mice that survived past 90 days revealed no evidence of tumor, suggesting eradication of established lesions in these long-term survivors. Immunohistochemical analysis of mice treated with SFV-IL12 documented an increased intratumoral infiltration of CD8 T cells (Fig. 4). This suggests the presence of the CTL response in vivo. 


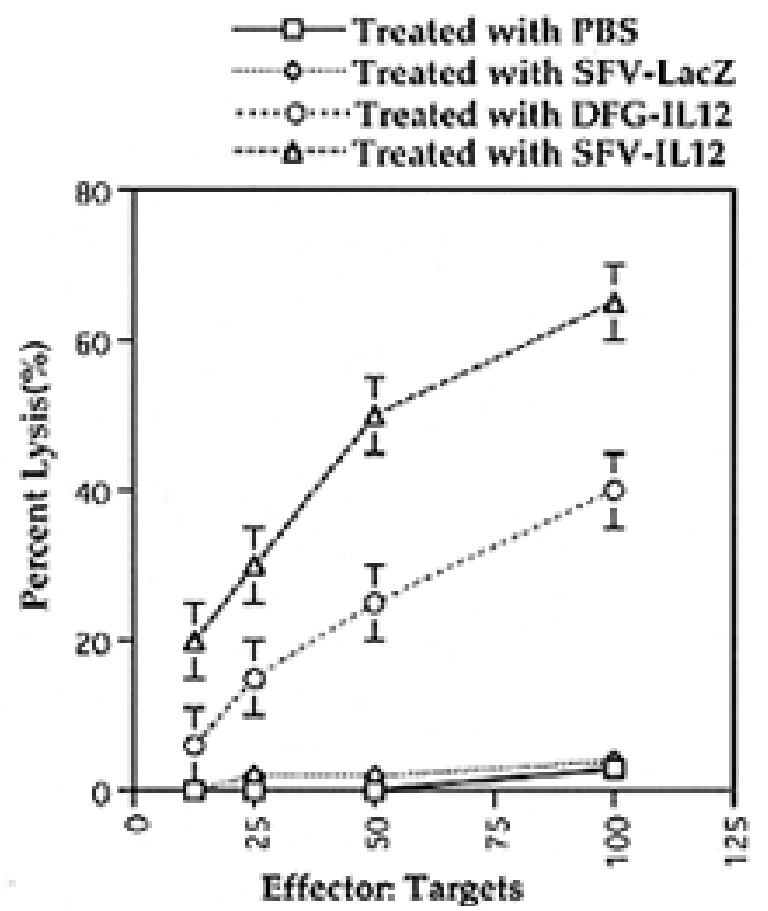

Fig. 2. Graph showing induction of specific lytic activity against tumor cells after immunization with SFV-IL12. Mice were immunized three times with either PBS, SFV-LacZ, DFG-IL12, and SFV-IL12. Seven days later, splenocytes were isolated and restimulated for 5 days with irradiated 203-glioma cells. Cytotoxic activity was then measured by $\mathrm{Cr}$ release assay by using glioma as the targets. Error bars indicate one standard deviation of the mean.

\section{Apoptosis Evident After Transfection With SFV and Uptake of SFV-Induced Apoptotic Cells by DCs}

After being transfected with SFV-IL12, cells rounded up, shrank, and stopped dividing after 24 hours. At 48 hours, these cells showed nuclear fragmentation characteristic of apoptosis (data not shown) . Nearly all of the cells were dead by 96 hours. The 203-glioma cells transfected with a conventional DNA plasmid encoding LacZ remained nearly as proliferative as nontransfected control cells (Fig. 5 upper). In the uptake experiment, cells were evaluated at 3 and 24 hours by using FACS analysis for PHK (red) and I-A ${ }^{\text {b }}$ (green). Double-positive cells were counted as a fraction of total FACS events. Uptake of SFV-induced apoptotic cells by DCs was also evident (Fig. 5 lower).

\section{DISCUSSION}

In this study, we demonstrated the prolongation of survival in tumor-bearing animals and induction of CTL response by using our vaccine strategy. The treatment of tumor-bearing mice with SFV-IL12 led to a dramatic reduction in the mortality rate at 90 days. At 90 days, $50 \%$ of the treated animals were still alive, and histological analysis indicated that SFV-IL12 therapy led to the eradication of established tumors in those long-term survivors. Systemic immune responses, as demonstrated by CTL activity, were significantly higher and tumor specific when SFV-IL12 was used.

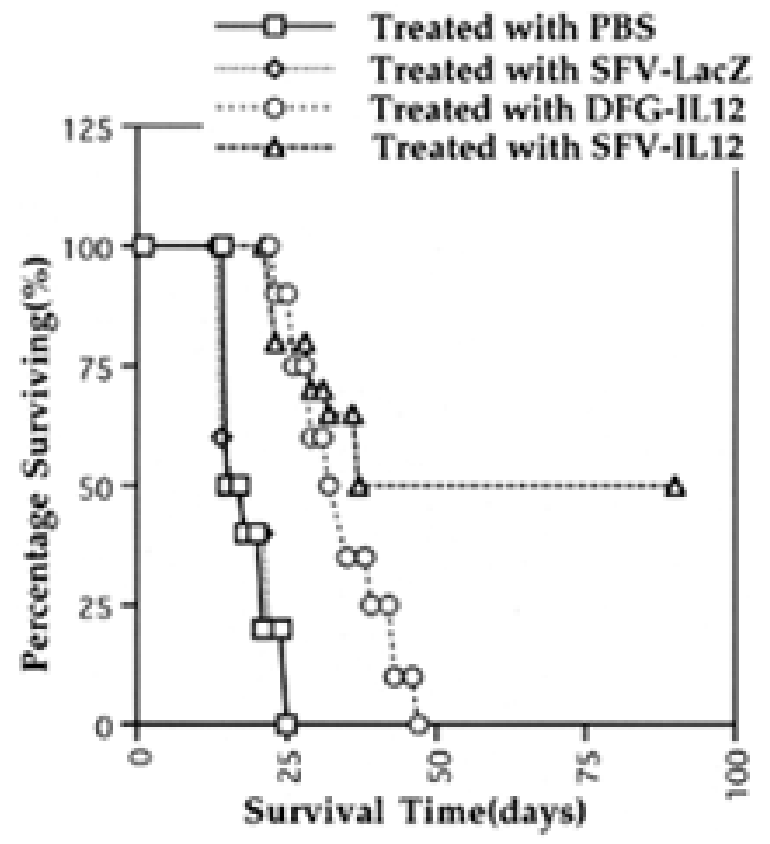

Fig. 3. Graph showing that the treatment of established 203glioma with SFV-IL12 prolongs survival. Intracranial tumor challenge with glioma cells was performed, and 4 days later, the mice were treated with a total of three immunizations spaced 1 week apart. Mice were evaluated daily until death. Median survival, was as follows: PBS group (10 mice) 20 days; SFV-LacZ group (12 mice), 20 days; DFG-IL 12 group (12 mice), 35 days; and SFVIL12 group (14 mice), 90 days.

Recent advances in tumor immunology have made it possible to evaluate new cytokine-based anticancer therapies. ${ }^{6,22}$ Among many cytokines evaluated as anticancer agents, IL12 confers potent antitumor immunity in murine tumor models. ${ }^{4,17,20,26,29}$ Systemic administration of IL-12 also was associated with severe dose-dependent toxicity in patients during the first clinical trial. The localized transfer of cytokine genes may circumvent some of the toxicity of systemic IL-12 delivery and provide adequate local cytokine levels for immune cell activation. ${ }^{17,20,26}$ In a recent report the authors showed that IL-12 acts directly on DCs to promote nuclear localization of nuclear factor $\mathrm{kB}$ and primes DCs for IL-12 production. ${ }^{11}$ This explains why the $I L-12$ gene is more effective in tumor treatment application. Cytokine and chemokine production induced by IL-12 stimulation at the tumor site might be responsible for these effects through alteration of the tumor microenvironment. A number of individual cytokine genes have also been shown to be effective in gene therapy studies in mouse tumor systems.

Semliki Forest virus is being developed as a vector for expression of heterologous genes and has many advantages for expression vector systems. In SFV systems, because helper RNA does not contain a packaging signal, it will not form a defective interfering particle or be packaged with recombinant RNA. Furthermore, replication occurs entirely in the cytoplasm of the infected cells as an RNA molecule, without a DNA intermediate. ${ }^{24}$ This behavior is in contrast with retroviruses, which must enter the nucleus and integrate into the host genome to initiate 


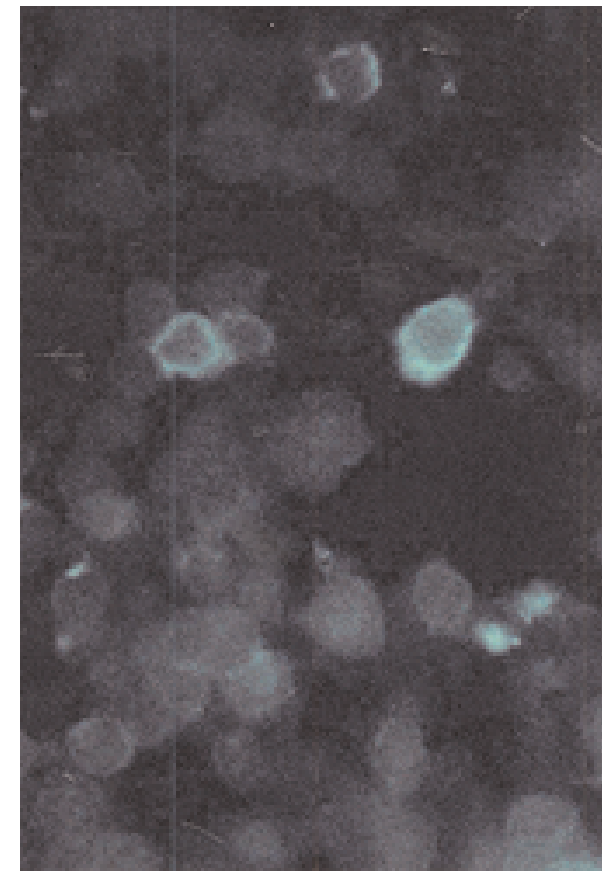

Fig. 4. Photomicrograph showing that 203-glioma vaccinated with SFV-IL12 demonstrates increased intratumoral infiltration of CD8 T cells. Intratumoral cells were evaluated using anti-CD8 antibody and fluorescein isothiocyanate-labeled antirat immunoglogulin $\mathrm{G}$ original magnification $\times 400$.

vector activity. Thus, retrovirus vectors have applications for long-term expression of foreign genes, whereas SFV is useful primarily for transient high-level expression. Furthermore, although adenovirus vectors can express high levels of foreign genes, these systems are more complex than SFV and express many highly antigenic virus-specific gene products including structural proteins. ${ }^{21}$ In contrast, current SFV systems express only the four viral replicase proteins (ns P1-4) required for RNA amplification in the transduced cells. The self-replicating RNA produced more than $200 \%$ the amount of antigen produced by a conventional DNA immunogen. ${ }^{33}$ This system has been found to express significant quantities of heterologous proteins in vitro ${ }^{13}$ and in vivo. ${ }^{19}$ As we have shown, DCs acquire antigen from apoptotic cells for further presentation to T cells. ${ }^{1,32}$ The SFV induces apoptosis in infected cells. ${ }^{14,33}$ Thus, it is possible that both processes, activation and apoptosis, as a result of the replicase activity upon SFV-IL12 infection, play an important syngeneic role in the initiation of specific immune responses against tumor cells. The other mechanisms underlying the enhanced immunogenicity is the enhanced uptake of antigen by DCs and other professional antigen-presenting cells. The results of this study suggest that intratumorally injected nontransduced immature DCs can acquire and process tumor antigens in situ, migrate to lymphoid organs, and initiate a significant tumor-specific immune response. In summary, we found that intratumoral injection with SFV-IL12 mediates an effective antitumor response that is superior to that observed in retroviral transduced IL-12 and is capable of inducing systemic antitumor immunity. Thus, the self-

\section{- - SFV transfected into 203 glioma \\ - Control plasmid transfected into 203 glioma}
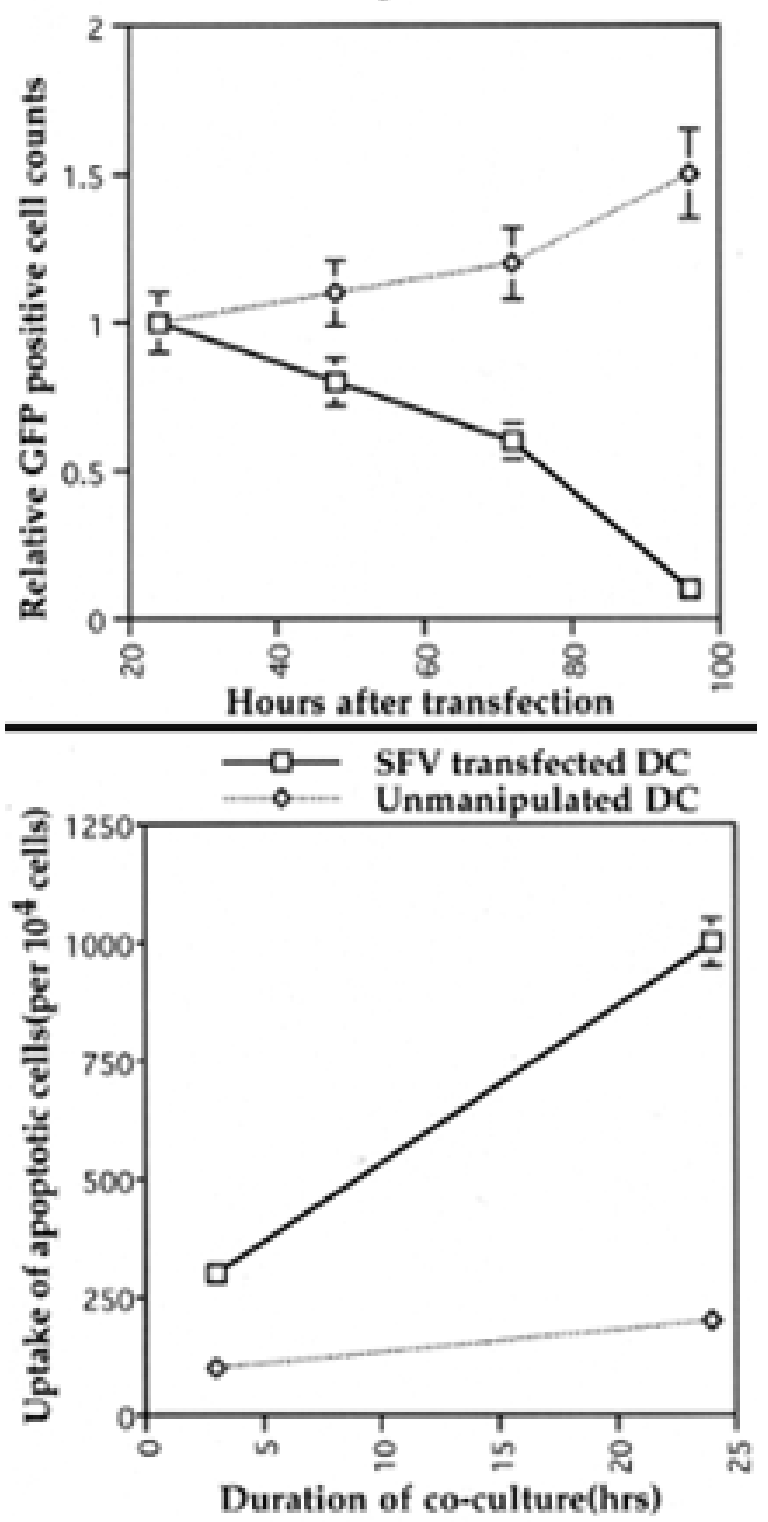

Fig. 5. Graphs showing induction of apoptotic death of host cells by SFV and its effect on the uptake by DCs. Upper: The SFV induces host cell death. The SFV-IL12 was transfected into 203-glioma cells; a control plasmid containing the LacZ gene under the control of the cytomegalovirus promoter was also transfected into glioma cells. Viable cells were counted every 24 hours to determine their survival. Lower: Uptake of SFV induced apoptotic cells by DCs. Unmanipulated or SFV-IL12-transfected glioma cells were labeled with PKH26-GL and then fed to immature DCs. Cells were evaluated at 3 and 24 hours by using FACS analysis for PHK and I-A $\mathrm{A}^{\mathrm{b}}$. Double-positive cells were counted as a fraction of total FACS events.

replicating SFV system could serve as a powerful tool in treating malignant brain tumors. 


\section{CONCLUSIONS}

The use of SFV-IL12 for the treatment of experimental brain tumors prolonged survival in tumor-bearing animals and induced CTL response. Thus, the self-replicating SFV system may be a novel approach for the treatment of malignant glioma.

\section{References}

1. Albert ML, Sauter B, Bhardwaj N: Dendritic cells acquire antigen from apoptotic cells and induce class I-restricted CTLs. Nature 392:86-89, 1998

2. Ashley DM, Faiola B, Nair S, et al: Bone marrow-generated dendritic cells pulsed with tumor extracts or tumor RNA induce antitumor immunity against central nervous system tumors. J Exp Med 186:1177-1182, 1997

3. Brunda MJ, Luistro L, Hendrzak JA, et al: Role of interferongamma in mediating the antitumor efficacy of interleukin-12. $\mathbf{J}$ Immunother Emphasis Tumor Immunol 17:71-77, 1995

4. Brunda MJ, Luistro L, Warrier RR, et al: Antitumor and antimetastatic activity of interleukin 12 against murine tumors. J Exp Med 178:1223-1230, 1993

5. Cui J, Shin T, Kawano T, et al: Requirement for Valpha14 NKT cells in IL-12-mediated rejection of tumors. Science 278: 1623-1626., 1997

6. Dilloo D, Bacon K, Holden W, et al: Combined chemokine and cytokine gene transfer enhances antitumor immunity. Nat Med 2:1090-1095, 1996

7. Engelhardt JF, Simon RH, Yang Y, et al: Adenovirus-mediated transfer of the CFTR gene to lung of nonhuman primates: biological efficacy study. Hum Gene Ther 4:759-769, 1993

8. Engelhardt JF, Yang Y, Stratford-Perricaudet LD, et al: Direct gene transfer of human CFTR into human bronchial epithelia of xenografts with E1-deleted adenoviruses. Nat Genet 4:27-34, 1993

9. Fakhrai H, Dorigo O, Shawler DL, et al: Eradication of established intracranial rat gliomas by transforming growth factor beta antisense gene therapy. Proc Natl Acad Sci USA 93: 2909-2914, 1996

10. Germann T, Rude E: Interleukin-12. Int Arch Allergy Immunol 108:103-112, 1995

11. Grohmann U, Belladonna ML, Bianchi R, et al: IL-12 acts directly on DC to promote nuclear localization of NF-kappaB and primes DC for IL-12 production. Immunity 9:315-323, 1998

12. Hsieh CS, Macatonia SE, Tripp CS, et al: Development of TH1 CD4+ T cells through IL-12 produced by Listeria-induced macrophages. Science 260:547-549, 1993

13. Levis R, Schlesinger S, Huang HV: Promoter for Sindbis virus RNA-dependent subgenomic RNA transcription. J Virol 64: 1726-1733, 1990

14. Lewis J, Wesselingh SL, Griffin DE, et al: Alphavirus-induced apoptosis in mouse brains correlates with neurovirulence. J Virol 70:1828-1835, 1996

15. Martinotti A, Stoppacciaro A, Vagliani M, et al: CD4 T cells inhibit in vivo the CD8-mediated immune response against murine colon carcinoma cells transduced with interleukin-12 genes. Eur J Immunol 25:137-146, 1995

16. Mulligan RC: The basic science of gene therapy. Science 260:926-932, 1993

17. Nastala CL, Edington HD, McKinney TG, et al: Recombinant IL-12 administration induces tumor regression in association with IFN-gamma production. J Immunol 153:1697-1706, 1994
18. Nishioka Y, Hirao M, Robbins PD, et al: Induction of systemic and therapeutic antitumor immunity using intratumoral injection of dendritic cells genetically modified to express interleukin 12. Cancer Res 59:4035-4041, 1999

19. Piper RC, Slot JW, Li G, et al: Recombinant Sindbis virus as an expression system for cell biology. Methods Cell Biol 43:55-78, 1994

20. Rakhmilevich AL, Turner J, Ford MJ, et al: Gene gun-mediated skin transfection with interleukin 12 gene results in regression of established primary and metastatic murine tumors. Proc Natl Acad Sci USA 93:6291-6296, 1996

21. Rosenfeld MA, Siegfried W, Yoshimura K,et al: Adenovirusmediated transfer of a recombinant alpha 1-antitrypsin gene to the lung epithelium in vivo. Science 252:431-434, 1991

22. Roth JA, Cristiano RJ: Gene therapy for cancer: what have we done and where are we going? J Natl Cancer Inst 89:21-39, 1997

23. Sampson JH, Ashley DM, Archer GE, et al: Characterization of a spontaneous murine astrocytoma and abrogation of its tumorigenicity by cytokine secretion. Neurosurgery 41:1365-1372, 1997

24. Strauss JH, Strauss EG: The alphaviruses: gene expression, replication, and evolution. Microbiol Rev 58:491-562, 1994

25. Tahara H, Lotze MT: Antitumor effects of interleukin-12 (IL12): applications for the immunotherapy and gene therapy of cancer. Gene Ther 2:96-106, 1995

26. Tahara H, Zeh HJ III, Storkus WJ, et al: Fibroblasts genetically engineered to secrete interleukin 12 can suppress tumor growth and induce antitumor immunity to a murine melanoma in vivo. Cancer Res 54:182-189, 1994

27. Tahara H, Zitvogel L, Storkus WJ, et al: Antitumor effects in patients with melanoma, head and neck and breast cancer in a Phase I/II clinical trial of interleukin-12 (IL-12). Proc Am Soc Clin Oncol 16:439, 1997 (Abstract)

28. Tahara H, Zitvogel L, Storkus WJ, et al: Effective eradication of established murine tumors with IL-12 gene therapy using a polycistronic retroviral vector. J Immunol 154:6466-6474, 1995

29. Tannenbaum CS, Wicker N, Armstrong D, et al: Cytokine and chemokine expression in tumors of mice receiving systemic therapy with IL-12. J Immunol 156:693-699, 1996

30. Tsung K, Meko JB, Peplinski GR, et al: IL-12 induces T helper 1-directed antitumor response. J Immunol 158:3359-3365, 1997

31. Yamanaka R, Tanaka R, Yoshida S, et al: Suppression of TGFbeta1 in human gliomas by retroviral gene transfection enhances susceptibility to LAK cells. J Neurooncol 43:27-34, 1999

32. Yamanaka R, Zullo SA, Tanaka R, et al: Genetically modified DC pulsed tumor cDNA via semliki forest virus markedly enhance antitumor immune responses in murine glioma models. J Neurosurg (In press, March issue, 2001)

33. Ying H, Zaks TZ, Wang RF, et al: Cancer therapy using a selfreplicating RNA vaccine. Nat Med 5:823-827, 1999

Manuscript received July 10, 2000.

Accepted in final form December 1, 2000.

Address reprint requests to: Ryuya Yamanaka, M.D., Department of Neurosurgery, Brain Research Institute, Niigata University, Asahimachi-dori 1-757, Niigata City 951-8122, Japan. email: ryaman@pop11.odn.ne.jp. 